Physics

Physics Research Publications

Purdue University

Year 2009

\title{
Compositional Contrast of Biological Materials in Liquids Using the Momentary Excitation of Higher Eigenmodes in Dynamic Atomic Force Microscopy
}
X. Xu
J. Melcher
S. Basak

R. Reifenberger

A. Raman

This paper is posted at Purdue e-Pubs.

http://docs.lib.purdue.edu/physics_articles/1065 


\title{
Compositional Contrast of Biological Materials in Liquids Using the Momentary Excitation of Higher Eigenmodes in Dynamic Atomic Force Microscopy
}

\author{
Xin Xu, ${ }^{1,3}$ John Melcher, ${ }^{1,3}$ Sudipta Basak, ${ }^{1,3}$ Ron Reifenberger, ${ }^{2,3}$ and Arvind Raman ${ }^{1,3}$ \\ ${ }^{1}$ School of Mechanical Engineering, Purdue University, West Lafayette, Indiana 47907, USA \\ ${ }^{2}$ Department of Physics, Purdue University, West Lafayette, Indiana 47907, USA \\ ${ }^{3}$ Birck Nanotechnology Center, Purdue University, West Lafayette, Indiana 47907, USA \\ (Received 10 July 2008; revised manuscript received 4 January 2009; published 13 February 2009)
}

Atomic Force microscope (AFM) cantilevers commonly used for imaging soft biological samples in liquids experience a momentary excitation of the higher eigenmodes at each tap. This transient response is very sensitive to the local sample elasticity under gentle imaging conditions because the higher eigenmode time period is comparable to the tip-sample contact time. By mapping the momentary excitation response, we demonstrate a new scanning probe spectroscopy capable of resolving with high sensitivity the variations in the elasticity of soft biological materials in liquids.

Dynamic atomic force microscopy (dAFM) is a versatile tool for the nanoscale imaging of biological membranes, viruses, proteins, cells, and intracellular structures in buffer solutions. dAFM methods for compositional contrast (elasticity or chemistry) in such soft materials are limited by the low cantilever quality factor in liquids [1-5]. In what follows, we demonstrate a significant improvement in this capability.

The nonlinear tip-sample interaction in dAFM induces cantilever vibrations at higher harmonics of the drive frequency $[1,5-10]$ which can provide compositional contrast $[1,5-7,9,11-13]$. Under ambient or vacuum conditions, the higher harmonics are very small and need to be enhanced using special cantilevers [12] or bimodal excitation [14]. However, in liquids, the higher harmonics are significantly larger, and the second harmonic has been used to map contrasts in local elasticity [5].

In this Letter, we build on recent work [10] to show that the tapping dynamics of soft AFM cantilevers in liquids features a unique nonlinear transient phenomenon - that of momentary excitation of higher eigenmodes which occurs naturally and does not require higher eigenmode frequencies to be integer multiples of the fundamental. We find these vibrations provide an order of magnitude improvement in compositional contrast on soft biological materials in liquids when compared to second harmonic imaging [5], under gentle imaging conditions and without the use of specialized cantilevers or intentional bimodal excitation $[12,14]$.

It is instructive to measure the motion of a soft rectangular microcantilever driven magnetically at its fundamental eigenmode when it interacts with a mica surface in a buffer solution under gentle imaging conditions, as shown in Fig. 1(a). The experimental deflection data from the photodiode position detector in an Agilent 5500 AFM system are acquired at $2.5 \mathrm{MHz}$ using National Instruments 5911 boards [15]. The distinct distortions seen in the wave- forms when the tip contacts the sample are due to the momentary excitation of the second eigenmode [10]. This momentary excitation enhances specific higher harmonics of the drive frequency that lie in the vicinity of the 2 nd eigenmode frequency as seen in Fig. 1(b).

To account for this distortion, a two-mode mathematical model for the tapping dynamics of magnetically excited,
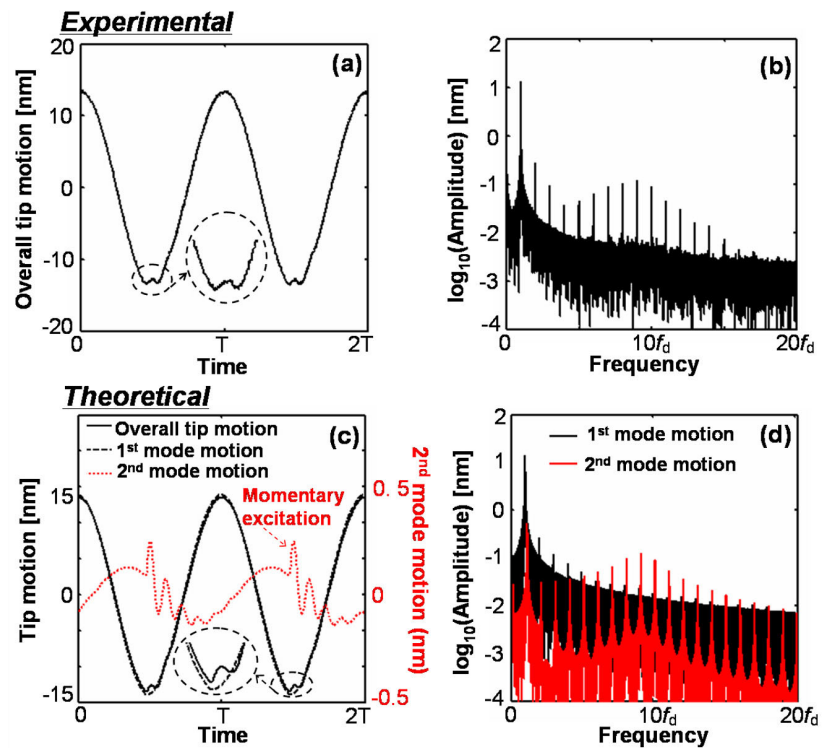

FIG. 1 (color online). (a) Experimental waveforms of cantilever deflection (cantilever properties listed in Ref. [15], sample preparation listed in [18]) tapping on mica in buffer solution with $14.1 \mathrm{~nm}$ initial amplitude at 95\% setpoint. (b) Discrete Fourier Transform (DFT) of the measured tip motion on mica demonstrating that the momentary excitation of the 2nd eigenmode results in the enhancement of specific higher harmonics of the drive frequency that lie in the vicinity of the 2 nd eigenmode resonance. (c) Simulated overall tip motion, 1st and 2nd eigenmode responses on mica for the same situation as in (a). (d) DFT of the theoretically predicted 1 st and 2 nd eigenmode motion. 
rectangular microcantilever is used [10]:

$$
\frac{\ddot{q}_{i}}{\omega_{i}^{2}}+\frac{\dot{q}_{i}}{\omega_{i} Q_{i}}+q_{i}=\frac{F_{i}}{k_{i}} \cos (\omega t)+\frac{F_{\mathrm{ts}}(z)}{k_{i}}
$$

where $i=1,2$ refers to the 1 st and 2 nd eigenmode, respectively, $q_{i}$ 's are the tip deflections in the $i$ th eigenmode, and dots represent temporal derivatives. $F_{i}, k_{i}, Q_{i}$, and $\omega_{i}$ are the equivalent forcing amplitudes, stiffnesses, quality factors, and natural frequencies of the $i$ th eigenmode, respectively, and $\omega$ (close to $\omega_{1}$ ) is the drive frequency. The tip-sample interaction force $F_{\mathrm{ts}}$ depends on the instantaneous gap between the tip and the sample $z=$ $Z_{c}+\left(q_{1}+q_{2}\right)$, where $Z_{c}$ is the equilibrium separation between the tip and the sample. Since any optical lever based technique senses the slope at the end of the cantilever, we plot the quantity $u=q_{1}+\chi q_{2}$, instead of the actual tip deflection $\left(q_{1}+q_{2}\right)$, where $\chi$ is the slope ratio of the 2nd and 1st eigenmodes at the cantilever's free end [16]. Using Hertzian contact mechanics [17,18]: $F_{\text {ts }}(z)=$ $\frac{4}{3} E^{*} \sqrt{R}(-z)^{3 / 2}$ for $z \leq 0$ and otherwise zero, where $R$ is the tip radius, $E^{*}$ is the effective elastic modulus of the tipsample system $\left(E_{\text {mica }}=60 \mathrm{GPa}, E_{\mathrm{Si}}=169 \mathrm{GPa}\right.$, Poisson ratios $=0.3$ ), MATLAB's ODE45 solver with finely time resolved steps is used to simulate Eq. (1).

Figure 1(c) shows the simulated tip motion $(u)$ and plots separately the 1st and 2nd eigenmode responses under the same situation as Fig. 1(a). The distortion at the bottom of the calculated waveform due to the momentary excitation and rapid decay of the 2nd eigenmode response are faithfully reproduced. The spectra of the simulated 1 st and 2 nd eigenmode motion are plotted in Fig. 1(d). The harmonic content attributed to the 1st eigenmode gradually diminishes as the harmonic number increases. By contrast, the harmonic content attributed to the momentary excitation (recurring once every drive cycle) contributes to a cluster of higher harmonics near the 2nd eigenmode natural frequency. For this specific cantilever, the 8th through 12th higher harmonics of the drive frequency are significantly enhanced due to momentary excitation and are henceforth referred to as momentary excitation (ME) harmonics. Because the momentary excitation occurs once every drive time period, it is periodic with respect to the drive frequency. It follows that the frequency spectrum of the momentary excitation signal contains only higher harmonics of the drive frequency.

One distinct advantage of the ME higher harmonics is the high sensitivity they exhibit to local material properties such as elasticity. We chose purple membrane (PM) for this study because it is a well-studied protein membrane that is easy to deposit with submonolayer coverage [19-23]. The sample preparation details are given in [18]. The experimental setup consists of an Agilent 5500 AFM system operating in the magnetic mode and an external Signal Recovery lock-in amplifier. The lock-in amplifier extracts the amplitudes of higher harmonics of the drive frequency for AFM imaging feedback, allowing higher harmonic images to be recorded simultaneously with normal topographic images over mica and PM $\left(E_{\text {mica }}=60 \mathrm{GPa}\right.$, $E_{\mathrm{PM}}=100 \mathrm{MPa}$ ). We focused on the extracellular face of PM which was identified by its surface roughness [21] in the topography images.

In a sequence of systematic experiments with soft cantilevers under gentle imaging conditions immersed in buffer solution, we first recorded the amplitude of the 1st through 15th harmonic signals simultaneously while performing feedback on the first harmonic. The resulting higher harmonic images are plotted in Fig. 2 [24]. The 9th harmonic image has the maximum contrast, which is an order of magnitude larger than the contrast of the 2nd harmonic. Indeed, a high degree of contrast is observed for the 8th through 12th harmonic (ME harmonic) images. Such re-
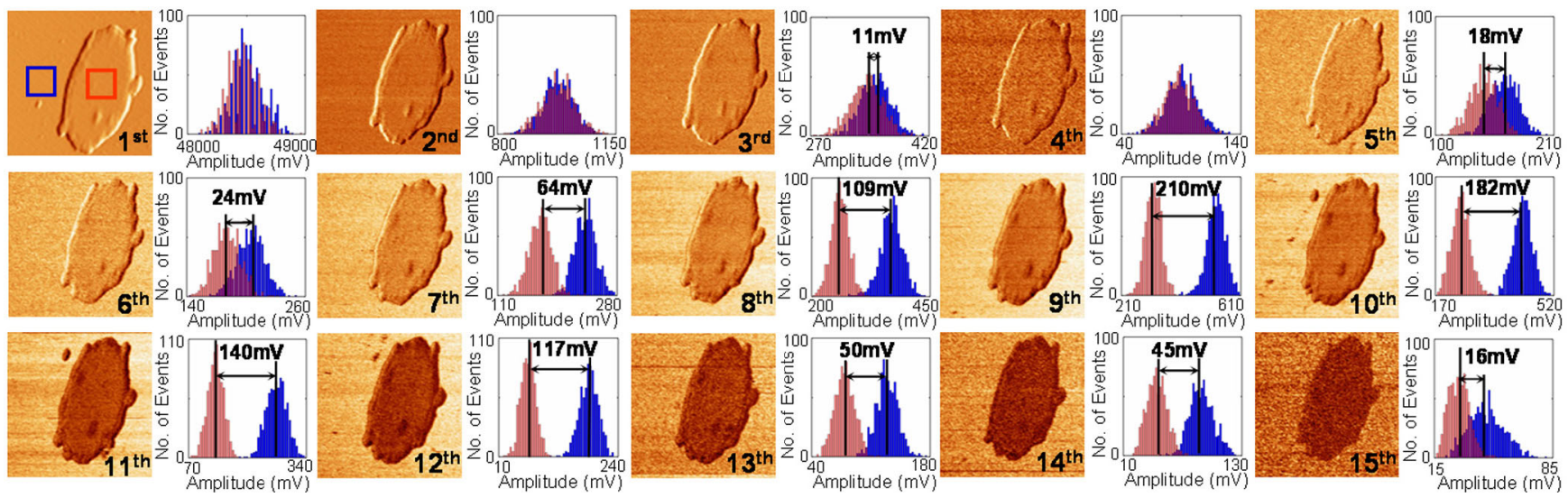

FIG. 2 (color online). Higher harmonic images $(1.3 \mu \mathrm{m} \times 1.3 \mu \mathrm{m})$ of a purple membrane (PM) patch on mica surface in buffer solution with corresponding histograms (15.4 nm initial amplitude, 97\% setpoint, cantilever properties listed in Ref. [15], sample preparation listed in [18]). The histograms are computed by taking a specific $220 \mathrm{~nm} \times 220 \mathrm{~nm}$ square area from the mica substrate and the PM patch respectively (see the inserted squares in the 1st harmonic panel). In the histograms [24], dark color is on mica and the light color is on PM. Each histogram is based on actual photodiode output at the specified harmonic. 
sults are consistently found for many soft cantilevers tested and are listed in the supplementary material [25].

We also measured the 2nd and 9th harmonic images of the extracellular faces of single and double layer purple membrane patches on a mica substrate (Fig. 3). According to Chadwick's theory for thin membranes on rigid substrate [26], the effect of doubling the membrane thickness is identical to reducing the sample stiffness by half. Remarkably, the 9th harmonic image in Fig. 3(c) clearly resolves the different stiffness of the single and double layers. By way of comparison, in the 2nd harmonic image shown in Fig. 3(b), no elasticity contrast is apparent.

It is important to keep in mind that while the local elastic modulus of PM can be sensitively affected by the $p \mathrm{H}$ and ionic concentration of the buffer solution [21,22], in our work all imaging is performed under identical high ionic concentration conditions [18]. Furthermore, we have used typical imaging conditions in liquids $(5-15 \mathrm{~nm}$ free amplitude) and magnetic cantilevers that provide moderate resolution images of PM. For high-resolution tapping mode images showing the molecular structure of bacteriorhodopsin, it becomes necessary to use very small free vibration amplitudes $(\sim 1 \mathrm{~nm})$ and oxide sharpened tips [23].

In order to better understand the mechanism of ME harmonic contrast, we describe a theory of the momentary excitation of the second eigenmode in liquids. First, it is important to realize that the tip-sample force impulse (integral of interaction force through contact time) is roughly independent of the material properties [27]. This allows a simplified interaction force model defined as $F_{\mathrm{ts}}^{*}=F_{\text {peak }}$ during tip-sample contact and otherwise zero. Peak force and contact time $t_{c}$ are related by a fixed tip-sample impulse $\hat{F}_{\text {ts }}^{*}=F_{\text {peak }} t_{c}$. Now, the second eigenmode will respond to this force impulse following the convolution integral $q_{2}^{*}(t)=\int_{0}^{t} F_{\mathrm{ts}}^{*}(\tau) h_{2}(t-\tau) d \tau, \quad$ where $h_{2}(t)=$
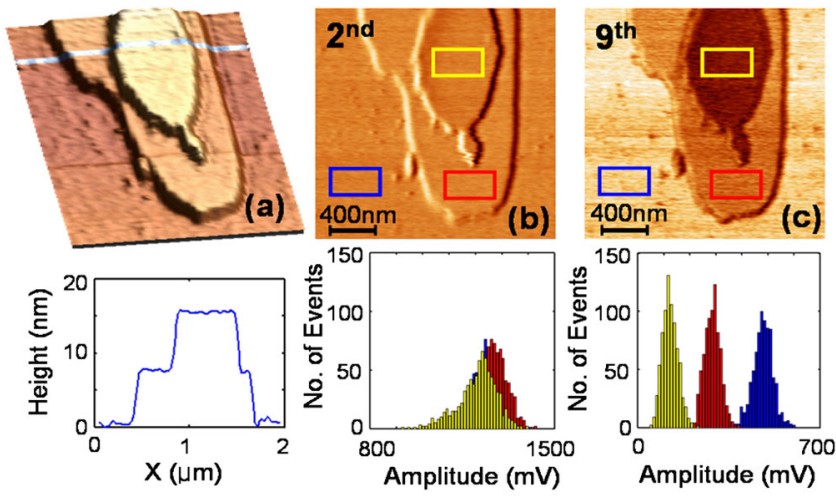

FIG. 3 (color online). Mica, single and double layer PM contrast in buffer solution (a) topography, (b) 2nd harmonic contrast, and (c) 9th harmonic contrast and corresponding profile and histograms (12.5 nm initial amplitude, 92\% setpoint, cantilever properties listed in Ref. [15], sample preparation listed in [18]). $\frac{\omega_{2}^{2}}{k_{2} \omega_{2 d}} e^{-\alpha t} \sin \omega_{2 d} t$ is the impulse response of the second eigenmode, $\omega_{2 d}=\omega_{2} \sqrt{1-1 / 4 Q_{2}^{2}}, \quad \alpha=\omega_{2} / 2 Q_{2}$, and $t=0$ marks the initiation of tip-sample contact. We can express the state of the second eigenmode $\left(q_{2}^{*}, \dot{q}_{2}^{*}\right)$ immediately after contact with the sample $\left(t=t_{c}\right)$ in terms of $t_{c}$ as follows

$$
\begin{gathered}
q_{2}^{*}\left(t_{c}^{-}\right)=\frac{\hat{F}_{\mathrm{ts}}^{*}}{k_{2} t_{c}}\left(1-e^{-\alpha t_{c}} \cos \frac{2 \pi t_{c}}{T_{2 d}}-\frac{\alpha T_{2 d}}{2 \pi} e^{-\alpha t_{c}} \sin \frac{2 \pi t_{c}}{T_{2 d}}\right) \\
\approx \frac{\hat{F}_{t s}^{*}}{k_{2} t_{c}}\left(1-\cos \frac{2 \pi t_{c}}{T_{2 d}}\right) \\
\dot{q}_{2}^{*}\left(t_{c}^{-}\right)=\frac{\hat{F}_{\mathrm{ts}}^{*} \omega_{2}^{2}}{2 \pi k_{2}} \frac{T_{2 d}}{t_{c}} e^{-\alpha t_{c}} \sin \frac{2 \pi t_{c}}{T_{2 d}}
\end{gathered}
$$

where the period of the second eigenmode is $T_{2 d}=$ $2 \pi / \omega_{2 d}$. For $t>t_{c}$, the second eigenmode rings down dictated by the initial state $q_{2}^{*}\left(t_{c}^{-}\right)$and $\dot{q}_{2}^{*}\left(t_{c}^{-}\right)$

$$
q_{2}^{*}(\bar{t})=e^{-\alpha \bar{t}}\left(c_{1} \sin \omega_{2 d} \bar{t}+c_{2} \cos \omega_{2 d} \bar{t}\right), \quad 0 \leq \bar{t} \leq T
$$

where, $\quad \bar{t}=t-t_{c}, \quad c_{2}=q_{2}^{*}\left(t_{c}^{-}\right), \quad$ and $\quad c_{1}=\left[\dot{q}_{2}^{*}\left(t_{c}^{-}\right)+\right.$ $\left.\alpha q_{2}^{*}\left(t_{c}^{-}\right)\right] / \omega_{2 d}$.

The above analysis offers two critical insights regarding momentary excitation of higher eigenmodes in liquids and its sensitivity to sample elasticity. For soft cantilevers in liquids under typical conditions, we expect imaging forces on the order of 1 nanonewton [28] and $k_{2}$ on the order of $10 \mathrm{~N} / \mathrm{m}$. Substituting $F_{\text {peak }}^{*}=\hat{F}_{\text {ts }}^{*} / t_{c}$ into Eqs. (2) and (3), we find that in liquids, the second eigenmode is momentarily excited with an initial amplitude of $\sim 0.1 \mathrm{~nm}$ and exponentially damps out before the next contact since $Q_{2}<\omega_{2} / \omega_{1}$. In air, however, stiff cantilevers are used so that $k_{2}$ is about 2 orders of magnitude greater and quality factors are high, resulting in very small amplitudes of the second eigenmode of $\sim 0.001 \mathrm{~nm}$ which do not damp out, as suggested by prior numerical studies [29].

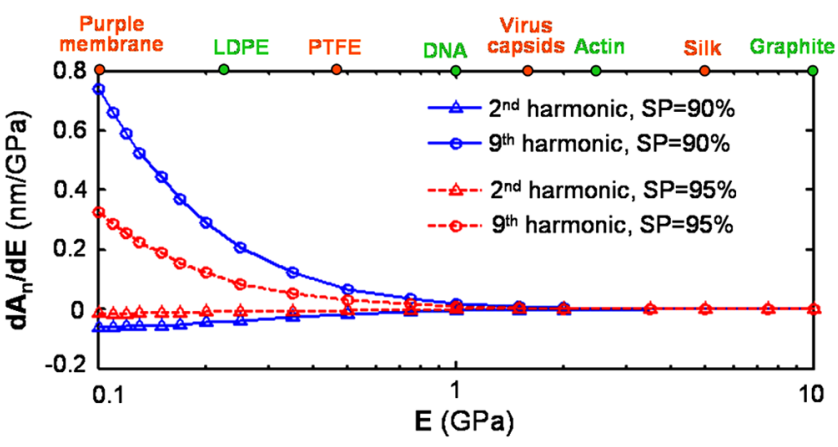

FIG. 4 (color online). Higher harmonic amplitude sensitivity to local sample elasticity $E$ for a $15 \mathrm{~nm}$ initial amplitude (cantilever properties listed in Ref. [15]) in liquid and using Eq. (1) with the Hertz contact model. 
Equations (2)-(4) demonstrate clearly that the momentary excitation is strongest when $t_{c}$ is of the same order as $T_{2 d}$, the time period of the second eigenmode, which naturally occurs under gentle imaging conditions (small $t_{c}$ ). Given that the contact time is governed primarily by elasticity, the momentary excitation of the second eigenmode becomes closely linked to the elasticity of the sample.

The sensitivity of different higher harmonics to local sample elasticity $E$ can be estimated from the change in the amplitude of the $n$th harmonic $\left(A_{n}\right)$ vs changes in $E$. Using Eq. (1) with a Hertz contact model and cantilever properties listed in [15], in Fig. 4 we plot $d A_{n} / d E$ of the 2nd and 9th ME harmonics vs $E$ for two amplitude setpoint ratios. Figure 4 illustrates that for setpoint amplitudes $>90 \%$, the ME harmonic amplitudes are (i) considerably more sensitive to elasticity than the 2nd harmonic, and (ii) the sensitivity to local material elasticity is greatest for $E<2 \mathrm{GPa}$. For lower setpoints, caution must be used to interpret higher harmonics due to the onset of multiple tapping regimes [27]. We conclude that for soft biological materials under gentle imaging conditions, the ME harmonics are about 1 order of magnitude more responsive than the 2 nd harmonic to local sample elasticity.

It is important to keep in mind that, in essence, momentary excitation is sensitive to short-range conservative forces. In this Letter, we designed experiments (soft membranes, high ionic concentration buffer) where these forces arise primarily from local elasticity allowing the use of momentary excitation to map local elasticity. However, it should be clear that experiments can be designed to exploit the momentary excitation to map other properties such as local electrostatic interactions or van der Waals forces of biological samples in buffer solutions.

By way of summary, we have shown that the momentary excitation of the second eigenmode is a naturally occurring phenomenon unique to AFM operation using soft cantilevers $\left(k_{1}<1 \mathrm{~N} / \mathrm{m}, Q_{1}<5\right)$ in liquids where quality factors are inherently low [10]. This phenomenon is manifest by the enhancement of integer higher harmonics of the drive frequency near the second eigenmode frequency and is quite sensitive to local elasticity when gentle imaging forces (high setpoints) are used. Taken together, this discovery provides a new scanning probe spectroscopy that significantly enhances elasticity contrast in soft biological samples under buffer solutions.

This research was supported by the National Science Foundation under Grant No. CMMI-0700289.

[1] S. J. T. Van Noort et al., Langmuir 15, 7101 (1999).

[2] T. Goodman et al., Langmuir 20, 3684 (2004).

[3] R. Gabai, L. Segev, and E. Joselevich, J. Am. Chem. Soc. 127, 11390 (2005).
[4] R. Garcia, R. Magerle, and R. Rerez, Nature Mater. 6, 405 (2007).

[5] J. Preiner et al., Phys. Rev. Lett. 99, 046102 (2007).

[6] R. Hillenbrand, M. Stark, and R. Guckenberger, Appl. Phys. Lett. 76, 3478 (2000).

[7] R. W. Stark and W. M. Heckl, Rev. Sci. Instrum. 74, 5111 (2003).

[8] J. Legleiter et al., Proc. Natl. Acad. Sci. U.S.A. 103, 4813 (2006).

[9] F. J. Giessibl, Surf. Interface Anal. 38, 1696 (2006).

[10] S. Basak and A. Raman, Appl. Phys. Lett. 91, 064107 (2007).

[11] A. Ulcinas and V. Snitka, Ultramicroscopy 86, 217 (2001).

[12] O. Sahin et al., Phys. Rev. B 69, 165416 (2004).

[13] S. Crittenden, A. Raman, and R. Reifenberger, Phys. Rev. B 72, 235422 (2005).

[14] J. R. Lozano and R. Garcia, Phys. Rev. Lett. 100, 076102 (2008).

[15] Properties of the cantilever used in Figs. 1-4 are: $k_{1}=$ $0.11 \mathrm{~N} / \mathrm{m}, Q_{1}=1.85, f_{1}=3.93 \mathrm{kHz}, k_{2}=8.05 \mathrm{~N} / \mathrm{m}$, $Q_{2}=4.29, f_{2}=30.00 \mathrm{kHz}$, and tip radius $R=50 \mathrm{~nm}$. Results with additional cantilevers are shown in the supplement. All cantilevers are driven at the frequency of the thermal peak of their fundamental eigenmode.

[16] For a rectangular cantilever with tip mass $=10 \%$ of the cantilever mass, standard beam theory predicts $\chi=4.59$.

[17] H. Hertz, J. Reine Angew. Math. 92, 156 (1882).

[18] In our experiments, wild type PM was diluted to $\sim 0.05 \mathrm{mg} / \mathrm{mL}$ in the imaging buffer $(300 \mathrm{mM} \mathrm{KCl}$, $20 \mathrm{mM}$ Tris-HCl, $p \mathrm{H} \mathrm{7.8)}$ and deposited onto freshly cleaved mica in a liquid cell. At these high ionic concentrations, the Debye length is sufficiently small $(\sim 0.5 \mathrm{~nm})$ that the electrostatic double layer force can be neglected. When the tip interacts with the sample surface, the repulsive elastic contact force is therefore the dominant interaction force.

[19] H. J. Butt, K. H. Downing, and P. K. Hansma, Biophys. J. 58, 1473 (1990).

[20] K. Voitchovsky et al., Biophys. J. 90, 2075 (2006).

[21] C. Moller et al., Biophys. J. 77, 1150 (1999).

[22] D. J. Muller et al., Biochim. Biophys. Acta 1460, 27 (2000).

[23] D. J. Muller and A. Engel, Nature Protocols 2, 2191 (2007).

[24] Note that the amplitude data are measured in Volts because two eigenmodes are involved in the response making it difficult to calibrate the amplitude measurement in units of nanometers.

[25] See EPAPS Document No. E-PRLTAO-102-020909. For more information on EPAPS, see http://www.aip.org/ pubservs/epaps.html.

[26] R. S. Chadwick, SIAM J. Appl. Math. 62, 1520 (2002).

[27] J. Melcher, X. Xu, and A. Raman, Appl. Phys. Lett. 93, 093111 (2008).

[28] X. Xu et al., Biophys. J. 95, 2520 (2008).

[29] T. R. Rodriguez and R. Garcia, Appl. Phys. Lett. 80, 1646 (2002). 